\title{
análisis cuantitativo por fluorescencia de rayos
}

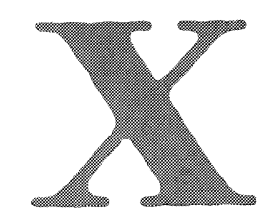

\section{J. L. SAGRERA, Ledo. en Ciencias Químicas}

Sección de Espectrometría del I. E. T. c. c.

\section{1. resumen}

Se describe en el presente trabajo el método operatorio para realizar un análisis cuantitativo rutinario de muestras naturales en estado pulverulento, determinando sus contenidos en calcio, silicio, aluminio, hierro, azufre y magnesio, expresados, todos ellos, en forma de óxidos.

Dentro de la misma publicación se hace referencia a trabajos en los cuales se explican, teórica y prácticamente, los procedimientos de preparación de muestras para el análisis de rayos $\mathrm{X}$ y eliminación de interferencias, aplicando la discriminación por altura de impulsos.

Las ventajas del método se especifican en un apartado del presente trabajo.

\section{2. descripción}

El análisis cuantitativo por fluorescencia de rayos $\mathrm{X}$ consiste en excitar la muestra $\mathrm{y}$ provocar una radiación fluorescente, la cual, una vez colimada, llega a un cristal analizador, donde se refleja según ángulos determinados, y se detecta en un contador, que lleva la respuesta a un registro gráfico o a un contador de impulsos.

El número de impulsos es proporcional a la concentración del elemento analizado, y por comparación con una muestra-patrón, cuyo análisis cuantitativo, efectuado por vía química, es conocido, se puede determinar la concentración de los elementos presentes en la muestra-problema, mediante una sencilla proporción aritmética.

\section{3. mbtodo operatorio}

Las muestras recibidas en el laboratorio son trituradas y molidas, hasta pasar toda la cantidad de muestra por el tamiz de 4.900 mallas.

Con el fin de eliminar el posible contenido de hierro metálico procedente de la molienda, 
a cada una de las muestras se le pasa un imán, quedando adherido al mismo las pequeñas partículas de aquel metal que se encuentre mezclado con la muestra.

Las muestras así obtenidas se desecan a $105^{\circ} \mathrm{C}$, hasta peso constante.

La pérdida al fuego de cada una de las muestras se determina por el procedimiento normal.

Con los datos de las pérdidas al fuego se forman grupos o familias, con pérdidas al fuego similares. Esta similitud se entiende para valores extremos que difieran en una unidad.

De cada familia se toma una muestra, cuya pérdida al fuego sea la media de los valores extremos que determina la familia. A esta muestra se le llama "patrón", analizándose sus componentes por la técnica química habitual y por duplicado.

Como es sabido, todo procedimiento de análisis por técnica fisicoquímica necesita un patrón, cuyo contenido haya sido determinado por vía química normal; dicho patrón se analizará también por la técnica fisicoquímica que se emplee. Esta determinación será el elemento comparativo de las muestras-problema de su misma familia.

Todas las muestras, tanto las patrones como las problema, son analizadas por fluorescencia de rayos X; para ello deben someterse a una preparación standard (1), que consiste esencialmente en tomar una cantidad aproximada de $10 \mathrm{~g}$, llevarlos a un portamuestras, y mediante un pistón cilíndrico se comprimen con una presión de $250 \mathrm{~kg} / \mathrm{cm}^{2}$ durante un tiempo fijo de medio minuto.

Una vez preparada la muestra, estará lista para ser introducida en el aparato de rayos $\mathrm{X}$ para su análisis.

El equipo utilizado para el presente trabajo tiene las características siguientes:

Marca: Philips.

Tipo: manual.

Unidad de alta tensión: tipo PW-1010.

Registrador: tipo PW-1051.

Goniómetro: tipo PW-1540.

Unidad de discriminación: PW-4072/01 y PW-4082.

En este tipo de aparato pueden analizarse simultáneamente cuatro muestras, de las cuales una de ellas será la tomada como patrón.

Como todo aparato sometido a cambios en la corriente de alimentación, los recuentos efectuados están influenciados por los cambios de la misma. Por ello, el método operatorio de recuentos tendrá que ser simultáneo para aminorar en lo posible sus influencias. Este tipo de determinación se realiza del modo siguiente: un recuento de la muestra-patrón, y un recuento de cada una de las tres muestras-problema. Un segundo recuento de la muestrapatrón y otro recuento de cada una de las muestras-problema anteriores. Esta operación constituye un ciclo que deberá repetirse cuatro o cinco veces, según sea la dispersión de los resultados en cada una de las muestras. Se calcula la media aritmética de los recuentos obtenidos para cada elemento. Se compara este resultado con el hallado con la muestrapatrón y se obtienen los resultados mediante una proporción aritmética. 
Las condiciones operatorias nara cada uno de los elementos determinados por rayos X son las siguientes:

a) Condiciones generales:

— ánodo de cromo trabajando a $40 \mathrm{kV}$ y $24 \mathrm{~mA}$;

- colimador ancho de $480 \mathrm{~mm}$;

- detector proporcional en flujo gaseoso a $1.630 \mathrm{~V}$.

b) Condiciones particulares:

- analizador de FLi para la determinación de hierro;

- analizador de A.D.P. para la determinación de magnesio;

- analizador de yeso para los restantes elementos;

- discriminación para el azufre:

- canal, $20 \mathrm{~V}$;

- atenuación, 2;

- amplitud, $30 \mathrm{~V}$;

- discriminación para el magnesio:

- canal, $16 \mathrm{~V}$;

- atenuación, 1 ;

- amplitud, $30 \mathrm{~V}$.

Los valores que se indican para las amplitudes corresponden, dada su variación con el tiempo de calefacción del aparato, a los utilizados al principio de los análisis.

Una explicación teórica y práctica de esta variación de la amplitud ha sido estudiada en un trabajo anterior (2).

A continuación se da una tabla de pérdidas al fuego, con expresión de las familias, precedidas por su patrón:

TABLA I

\begin{tabular}{c|c|c|c} 
Muestra & \multicolumn{2}{c}{ Pérdida al fuego } & \multicolumn{2}{c}{ Muestra } & Pérdida al fuego \\
Patrón 1 & 37,38 & & \\
45 & 37,69 & 70 & 38,39 \\
48 & 37,32 & 56 & 35,94 \\
4 & 37,36 & 53 & 36,61 \\
64 & 37,44 & 37 & 36,06 \\
78 & 37,44 & 22 & 36,16 \\
86 & 37,46 & Patrón 6 & 36,30 \\
67 & 37,47 & 58 & 35,79 \\
Patrón 3 & 38,41 & 54 & 35,66 \\
50 & 38,55 & 55 & 35,80 \\
41 & 38,55 & 23 & 35,32 \\
20 & 38,56 & 57 & 35,37 \\
79 & 38,36 & & 35,52
\end{tabular}


TABLA I (Continuación)

\begin{tabular}{|c|c|c|c|}
\hline Muestra & Pérdida al fuego & Muestra & Pérdida al fuego \\
\hline Patrón 12 & 17,34 & Patrón 35 & 37,98 \\
\hline 10 & 17,27 & 26 & 37,86 \\
\hline Patrón 14 & 14,44 & 61 & 37,91 \\
\hline 11 & 14,96 & Patrón 44 & 40,80 \\
\hline 18 & 14,17 & 59 & 40,25 \\
\hline Patrón 17 & 24,39 & 73 & 40,49 \\
\hline 13 & 24,97 & Patrón 52 & 39,97 \\
\hline Patrón 25 & 39,36 & 60 & 39,34 \\
\hline 80 & 39,02 & 8 & 39,41 \\
\hline 68 & 39,15 & 9 & 39,49 \\
\hline Patrón 31 & 23,27 & Patrón 69 & 38,97 \\
\hline 28 & 23,15 & 49 & 38,61 \\
\hline 29 & 23,76 & 74 & 38,71 \\
\hline 30 & 22,99 & 51 & 38,77 \\
\hline Patrón 32 & 21,21 & 72 & 38,93 \\
\hline 33 & 21,24 & 47 & 38,67 \\
\hline Patrón 34 & 39,84 & 21 & 39,08 \\
\hline 62 & . $\quad 39,47$ & Patrón 76 & 37,26 \\
\hline 19 & 39,53 & 27 & 36,69 \\
\hline 39 & 39,76 & 88 & 36,87 \\
\hline Patrón 35 & 37,98 & 42 & 37,03 \\
\hline 46 & 38,06 & 36 & 37,18 \\
\hline 87 & 37,69 & Patrón 77 & 38,19 \\
\hline 63 & 37,76 & 5 & 37,90 \\
\hline 40 & 37,77 & 81 & 38,00 \\
\hline 66 & 37,78 & Patrón 82 & 38,56 \\
\hline \multirow[t]{4}{*}{65} & 37,80 & 85 & 38,15 \\
\hline & & 83 & 38,27 \\
\hline & & 71 & 38,41 \\
\hline & & 38 & 38,43 \\
\hline Patrón 16 & 28,46 & Patrón 15 & 32,57 \\
\hline Patrón 2 & 38,55 & Patrón 75 & 38,73 \\
\hline Patrón 43 & 40,59 & Patrón 84 & 43,28 \\
\hline Patrón 24 & 33,94 & & \\
\hline
\end{tabular}

Los siete últimos patrones no pertenecen a ninguna familia; se han utilizado solamente como comprobación. 
Como se ha expuesto cada uno de los patrones ha sido analizado por vía química. En la tabla que sigue se expresan los resultados de los análisis de dichos patrones:

TABLA II

\begin{tabular}{rrrrrrr} 
Muestra & $\mathrm{SiO}_{2}$ & $\mathrm{Al}_{2} \mathrm{O}_{3}$ & $\mathrm{Fe}_{2} \mathrm{O}_{3}$ & $\mathrm{CaO}$ & $\mathrm{MgO}$ & $\mathrm{SO}_{3}$ \\
\hline 1 & 9,80 & 3,30 & 1,38 & 46,80 & 0,53 & 0,06 \\
2 & 8,19 & 2,49 & 1,16 & 48,40 & 0,62 & 0,05 \\
3 & 7,96 & 2,50 & 1,02 & 48,52 & 0,64 & 0,19 \\
6 & 12,45 & 3,59 & 1,36 & 45,19 & 0,60 & 0,12 \\
7 & 12,45 & 4,02 & 1,42 & 44,65 & 0,60 & 0,18 \\
12 & 42,12 & 12,22 & 5,01 & 17,10 & 3,10 & 0,52 \\
14 & 48,11 & 14,22 & 5,28 & 11,43 & 3,30 & 0,95 \\
15 & 17,07 & 5,49 & 2,18 & 39,90 & 1,37 & 0,49 \\
16 & 23,78 & 8,02 & 2,79 & 33,30 & 1,69 & 0,43 \\
17 & 30,55 & 9,93 & 3,49 & 27,15 & 1,97 & 0,50 \\
24 & 15,39 & 4,77 & 2,36 & 41,10 & 1,42 & 0,14 \\
25 & 6,95 & 1,71 & 0,86 & 49,70 & 0,86 & 0,15 \\
31 & 32,31 & 10,39 & 3,46 & 26,26 & 1,90 & 0,65 \\
32 & 35,64 & 11,57 & 3,48 & 22,98 & 2,01 & 0,80 \\
34 & 5,89 & 2,13 & 0,87 & 50,30 & 0,46 & 0,04 \\
35 & 8,49 & 3,23 & 1,35 & 47,70 & 0,56 & 0,13 \\
43 & 4,74 & 1,73 & 0,87 & 51,50 & 0,21 & 0,08 \\
44 & 4,24 & 1,60 & 0,78 & 51,80 & 0,24 & 0,00 \\
52 & 5,75 & 2,04 & 0,96 & 50,57 & 0,46 & 0,00 \\
69 & 6,45 & 2,93 & 1,26 & 49,10 & 0,41 & 0,00 \\
75 & 6,99 & 2,60 & 1,14 & 49,40 & 0,59 & 0,05 \\
76 & 9,73 & 3,99 & 1,59 & 46,40 & 0,54 & 0,00 \\
77 & 8,27 & 3,16 & 1,38 & 47,82 & 0,63 & 0,00 \\
82 & 7,51 & 3,22 & 1,39 & 48,54 & 0,33 & 0,00 \\
84 & 0,43 & 0,15 & 0,18 & 55,54 & 0,24 & 0,00 \\
& & & & & &
\end{tabular}

La media aritmética obtenida mediante fluorescencia de rayos $\mathrm{X}$ en la muestra-patrón, estará en la misma proporción con su porcentaje del elemento a determinar que las medias aritméticas obtenidas en las muestras-problema con sus contenidos a determinar. Es decir:

$$
\frac{\text { Recuento de la muestra-patrón }}{\text { Porcentaje del elemento }}=\frac{\text { Recuento de la muestra-problema }}{\mathrm{X} \% \text { del elemento }}
$$


En la siguiente tabla se dan los resultados finales del análisis de las muestras-problema, determinados por fluorescencia de rayos $\mathrm{X}$ :

\begin{tabular}{|c|c|c|c|c|c|c|c|}
\hline \multicolumn{8}{|c|}{ TABLA III } \\
\hline Muestra & $\mathrm{SiO}_{2}$ & $\mathrm{Al}_{2} \mathrm{O}_{3}$ & $\mathrm{Fe}_{2} \mathrm{O}_{3}$ & $\mathrm{CaO}$ & $\mathrm{MgO}$ & $\mathrm{SO}_{3}$ & Total \\
\hline 45 & 8,61 & 3,15 & 1,43 & 48,40 & 0,43 & 0,06 & 100,31 \\
\hline 48 & 9,39 & 3,25 & 1,30 & 47,21 & 0,42 & 0,06 & 99,53 \\
\hline 4 & 9,23 & 3,19 & 1,09 & 47,48 & 0,73 & 0,06 & 99,80 \\
\hline 64 & 8,79 & 3,25 & 1,97 & 48,62 & 0,53 & 0,06 & 100,56 \\
\hline 78 & 8,43 & 3,12 & 1,31 & 48,32 & 0,44 & 0,06 & 99,73 \\
\hline 86 & 8,17 & 3,08 & 1,39 & 49,09 & 0,43 & 0,06 & 100,22 \\
\hline 67 & 8,36 & 3,13 & 1,34 & 48,46 & 0,41 & 0,06 & 99,85 \\
\hline 50 & 7,48 & 2,50 & 1,25 & 49,03 & 0,42 & 0,18 & 99,91 \\
\hline 20 & 7,98 & 2,54 & 1,17 & 48,38 & 0,50 & 0,19 & 99,64 \\
\hline 79 & 7,33 & 2,47 & 1,25 & 49,26 & 0,48 & 0,19 & 99.81 \\
\hline 70 & 8,02 & 2,58 & 1,34 & 48,39 & 0,48 & 0,19 & 99,85 \\
\hline 41 & 7,66 & 2,49 & 1,23 & 48,74 & 0,48 & 0,19 & 99,84 \\
\hline 56 & 12,22 & 3,73 & 1,49 & 45,26 & 0,58 & 0,11 & 100,50 \\
\hline 53 & 11,91 & 3,51 & 1,36 & 45,82 & 0,56 & 0,13 & 99,93 \\
\hline 37 & 12,17 & 3,42 & 1,20 & 45,93 & 0,49 & 0,12 & 100,15 \\
\hline 22 & 11,97 & 3,90 & 1,79 & 45,42 & 0,39 & 0,10 & 100,47 \\
\hline 58 & 13,20 & 4,04 & 1,79 & 43,24 & 0,71 & 0,17 & 99,44 \\
\hline 54 & 13,04 & 3,97 & 1,53 & 44,00 & 0,71 & 0,17 & 99,90 \\
\hline 55 & 13,34 & 4,00 & 1,76 & 42,88 & 0,73 & 0,15 & 98,78 \\
\hline 23 & 13,32 & 4,22 & 2,18 & 42,71 & 0,73 & 0,15 & 99,34 \\
\hline 57 & 12,91 & 3,99 & 1,78 & 43,43 & 0,71 & 0,15 & 99,22 \\
\hline 10 & 44,30 & 12,37 & 5,12 & 16,09 & 3,23 & 0,48 & 101,34 \\
\hline 11 & 46,78 & 14,23 & 5,41 & 11,59 & 3,43 & 1,01 & 100,03 \\
\hline 18 & 48,16 & 14,31 & 5,28 & 11,39 & 3,49 & 0,92 & 100,46 \\
\hline 13 & 31,17 & 9,26 & 3,30 & 28,48 & 1,49 & 0,50 & 100,61 \\
\hline 80 & 6,29 & 1,78 & 1,18 & 49,41 & 0,65 & 0,14 & 98,99 \\
\hline 68 & 6,17 & 1,73 & 1,22 & 49,76 & 0,65 & 0,15 & 99,24 \\
\hline 28 & 30,44 & 10,53 & 3,40 & 27,41 & 1,85 & 0,71 & 99,24 \\
\hline 29 & 29,62 & 9,97 & 3,07 & 29,60 & 1,71 & 0,79 & 100,26 \\
\hline 30 & 32,93 & 10,56 & 3,63 & 25,46 & 1,75 & 0,64 & 99,58 \\
\hline 33 & 36,23 & 11,75 & 3,56 & 22,54 & 2,05 & 0,74 & 100,17 \\
\hline 62 & 5,92 & 2,09 & 0,82 & 50,07 & 0,42 & 0,04 & 99,19 \\
\hline 19 & 5,92 & 2,07 & 0,88 & 49,39 & 0,49 & 0,04 & 98,70 \\
\hline 39 & 5,61 & 2,05 & 0,77 & 50,32 & 0,48 & 0,04 & 99,51 \\
\hline 46 & 8,31 & 3,30 & 1,43 & 48,52 & $0, \overline{5} 4$ & 0,11 & 100,84 \\
\hline 87 & 8,37 & 3,26 & 1,44 & 47,83 & 0,56 & 0,12 & 99,83 \\
\hline 63 & 8,36 & 3,30 & 1,44 & 47,99 & 0,54 & 0,12 & 100,01 \\
\hline 40 & 8,83 & 3,32 & 1,46 & 47,20 & 0,51 & 0,13 & 99,70 \\
\hline 66 & 8,27 & 3,25 & 1,47 & 48,45 & 0,56 & 0,12 & 100,48 \\
\hline 65 & 8,31 & 3,23 & 1,31 & 48,45 & 0,54 & 0,11 & 100,31 \\
\hline 26 & 9,00 & 3,37 & 1,51 & 47,60 & 0,64 & 0,13 & 100,79 \\
\hline 61 & 8,51 & 3,28 & 1,39 & 48,61 & 0,53 & 0,12 & 100,81 \\
\hline 59 & 4,35 & 1,61 & 0,73 & 52,17 & 0,25 & 0,00 & 99,80 \\
\hline 73 & 4,19 & 1,61 & 0,71 & 52,48 & 0,25 & 0,00 & 99,99 \\
\hline
\end{tabular}


TABLA III (Continuación)

\begin{tabular}{|c|c|c|c|c|c|c|c|}
\hline Muestra & $\mathrm{SiO}_{2}$ & $\mathrm{Al}_{2} \mathrm{O}_{3}$ & $\mathrm{Fe}_{2} \mathrm{O}_{3}$ & $\mathrm{CaO}$ & $\mathrm{MgO}$ & $\mathrm{SO}_{3}$ & Total \\
\hline 60 & 5,95 & 2,06 & 1,12 & 50,12 & 0,41 & 0,00 & 99,37 \\
\hline 8 & 5,84 & 2,10 & 1,11 & 49,96 & 0,43 & 0,00 & 99,15 \\
\hline 9 & 5,93 & 2,08 & 1,05 & 49,52 & 0,43 & 0,00 & 98,80 \\
\hline 49 & 6,64 & 3,00 & 1,20 & 49,58 & 0,43 & 0,00 & 99,86 \\
\hline 74 & 6,57 & 2,40 & 1,20 & 49,55 & 0,48 & 0,00 & 99,33 \\
\hline 51 & 7,37 & 2,60 & 0,98 & 48,90 & 0,50 & 0,00 & 99,49 \\
\hline 72 & 6,99 & 2,49 & 1,09 & 49,62 & 0,67 & 0,00 & 100,25 \\
\hline 47 & 6,76 & 2,99 & 1,09 & 49,81 & 0,49 & 0,00 & 100,38 \\
\hline 21 & 6,47 & 2,95 & 1,00 & 49,55 & 0,47 & 0,00 & 99,96 \\
\hline 27 & 10,12 & 4,18 & 1,74 & 45,04 & 0,56 & 0,00 & 99,07 \\
\hline 88 & 9,55 & 3,89 & 1,54 & 46,56 & 0,58 & 0,00 & 99,65 \\
\hline 42 & 9,61 & 3,95 & 1,65 & 46,13 & 0,58 & 0,00 & 99,54 \\
\hline 36 & 10,37 & 3,84 & 1,15 & 46,93 & 0,71 & 0,00 & 100,81 \\
\hline 5 & 8,97 & 3,16 & 1,10 & 47,32 & 0,98 & 0,00 & 99,93 \\
\hline 81 & 8,28 & 3,27 & 1,23 & 47,65 & 0,66 & 0,00 & 99,77 \\
\hline 85 & 7,64 & 3,19 & 1,19 & 48,12 & 0,35 & 0,00 & 99,12 \\
\hline 83 & 7,49 & 3,20 & 1,30 & 49,25 & 0,35 & 000 & 99,36 \\
\hline 71 & 8,01 & 3,11 & 1,24 & 48.41 & 0,46 & 0,00 & 100,12 \\
\hline 38 & 7,90 & 3,24 & 1,15 & 48,34 & 0,35 & 0,00 & 99,85 \\
\hline
\end{tabular}

\section{4. conclusiones}

A la vista de la tabla III del presente trabajo, se comprueba que, a pesar de la diversidad en los contenidos de las muestras-problema, los resultados suman cien en su mayoría. Teniendo en cuenta que los resultados de rayos $\mathrm{X}$ están afectados por los posibles errores de las determinaciones analíticas por vía química, la técnica expuesta puede considerarse aceptable.

El número de patrones que han tenido que ser analizados por vía química en el presente trabajo es elevado, debido a la variedad de la composición de las muestras.

$\mathrm{Si}$ se tiene en cuenta que los porcentajes de $\mathrm{Na}_{2} \mathrm{O}$ y $\mathrm{K}_{2} \mathrm{O}$ se han obtenido mediante otra técnica analítica, la suma de los resultados hallados también estará afectada por el error inherente a esta técnica.

$\mathrm{El}$ método de análisis por fluorescencia de rayos $\mathrm{X}$ tiene las ventajas siguientes:

a) es no destructivo, puesto que la muestra se recupera una vez efectuado el análisis;

b) es rápido, ya que en la determinación de cada elemento se requieren de tres a seis minutos;

c) el manejo del aparato puede realizarlo cualquier persona no especializada, en condiciones de trabajo standard;

d) el entretenimiento del equipo de rayos $\mathrm{X}$ es barato $\mathrm{y}$ sencillo contra la manutención de todo laboratorio químico.

\section{5. bibliografia}

(1) Triviño, Sagrera, Calleja: "La preparación de muestras para análisis por rayos X". I Fluorescencia. Ultimos Avances en Materiales de Construcción, núm. 113, enero-febrero-marzo 1964.

(2) Triviño, Sagrera: “Discriminación por altura de impulsos”. Monografía, núm. 242, del I.E.T.c.c. Madrid, 1964. 